\title{
Migración Criminal en Zonas Turísticas Mexicanas
}

\section{Criminal Migration in Mexican Tourist Zones}

DOI: $10.46932 / \operatorname{sfjdv3n1-080}$

Received in: Jan 30st, 2021

Accepted in: Feb 1th, 2022

\author{
Martha Fabiola García-Álvarez \\ Doctor in Law, Altos University Centre, University of Guadalajara; av. Rafael Casillas Aceves no. \\ 1200, Tepatitlán de Morelos, Jalisco, México \\ E-mail: comienzofabiola.2018@gmail.com
}

\section{Carla Monroy-Ojeda}

Doctor in Criminology, University of Security Sciences of the State of Nuevo Leon, Santa Dorotea, 355, col. Fuentes de Santa Lucía, cp. 66647, Apodaca, Nuevo León, México

E-mail: crlmonroy@gmail.com

\section{Dante Jaime Haro-Reyes}

Doctor in law, University Centre of Social Sciences and Humanities, University of Guadalajara; Av. Lerdo de Tejada 2640, col. Arcos Vallarta, cp. 44130, Guadalajara, Jalisco, México

E-mail: dante.haro@redudg.udg.mx

\section{Ramón Gerardo Navejas-Padilla}

Doctor en Educación, University Centre of Los Valles, University of Guadalajara; Carretera

Guadalajara- Ameca km 45.5, cp. 46600, 3331917930

E-mail: ramón.navejas@academicos.udg.mx

\section{RESUMEN}

La migración y movilidad poblacional es un fenómeno que, con las crecientes relaciones globales y facilidades para el desplazamiento, han aumentado considerablemente. Esto se observa en el día a día, en todos los ámbitos humanos como, por ejemplo, las actividades turísticas, en donde hay desplazamiento de los propios visitantes, como de aquellas personas que buscan ofertas de trabajo para mejorar sus condiciones de vida. En este sentido, se conjugan diferentes factores como lo son, grupos vulnerables, turismo sexual, migración, redes criminales, cultura patriarcal y de violencia, que hace que México sea, no sólo un destino con lugares afrodisíacos, sino un lugar en de alta inseguridad para grupos vulnerables que son traficados y con imperiosa necesidad para detener tales prácticas. De acuerdo a la Organización Internacional de las Migraciones, México ocupa el segundo lugar en turismo sexual infantil. Cancún, Acapulco, Guadalajara, Tapachula y Tijuana, son las principales ciudades en donde se permiten tales prácticas. La relación es que, son ciudades de tradición y atracción turística importante, son cruces fronterizos (Tapachula y Tijuana), y en donde las organizaciones criminales tienen una fuerte presencia. En este sentido, es importante conocer el perfil geográfico de aquellas personas que migran para cometer delitos, siendo una forma de violencia y donde existen víctimas. En este trabajo, que es parte de un proyecto de investigación, está basado en los Objetivos del Milenio de la ONU, dentro del Eje 16 "Promover sociedades pacíficas e inclusivas para el desarrollo sostenible, facilitar acceso a la justicia para todos..."; así como, dentro del marco del Programa Nacional Estratégico (PRONACES) del CONACYT, en la línea de investigación violencias estructurales. Son necesarias políticas públicas y criminales, 
estrategias programas y acciones para la detección y posibilidad de implementar la respectiva prevención hacia delincuentes o en riesgo de delinquir, y protección a las víctimas vulnerables.

Palabras Clave: Migración, redes criminales, turismo sexual, vulnerabilidad, violencia.

\begin{abstract}
Population migration and mobility is a phenomenon that, with increasing global relations and ease of displacement, has increased considerably. This is observed on a daily basis, in all human fields, such as tourist activities, where there is displacement of the visitors themselves, as well as those who seek job opportunities to improve their living. In this sense, different factors are combined, such as vulnerable groups, sex tourism, migration, criminal networks, A patriarchal culture of violence, which makes Mexico not only a destination with aphrodisiac places, but a place of high insecurity for vulnerable groups who are trafficked and with urgent need to stop such practices. According to the International Organization for Migration, Mexico ranks second in child sex tourism. Cancun, Acapulco, Guadalajara, Tapachula and Tijuana are the main cities where such practices are permitted. The relationship is that, they are cities of tradition and major tourist attraction, they are border crossings (Tapachula and Tijuana), and where criminal organizations have a strong presence. In this regard, it is important to know the geographical profile of those who migrate to commit crimes, being a form of violence and where there are victims. This work, which is part of a research project, is based on the UN Millennium Goals, under Axis 16 "Promoting peaceful and inclusive societies for sustainable development, facilitating access to justice for all..."; As well as, within the framework of the National Strategic Program (PRONACES) of CONACYT, in the line of research structural violence. Public and criminal policies, strategies programs and actions are needed to detect and implement the respective prevention of offenders or offenders at risk of crime, and protection of vulnerable victims.
\end{abstract}

Keywords: Migration, criminal networks, sex tourism, vulnerability, violence.

\title{
1 INTRODUCCIÓN
}

El presente trabajo adentrará al lector a una perspectiva y análisis sobre la movilidad criminal en zonas turísticas mexicanas, formas en que las personas migras, y razones que tienen para hacerlo; así como puntos clave dentro de la República Mexicana, a donde convergen generalmente los que migran de manera internan y externa.

Asimismo, se detallan los factores que interviene para que exista ese desplazamiento, tales como la vulnerabilidad y violencia de que son objeto las víctimas y, cómo es la dinámica que prevalece por parte de las Redes Criminales, para capturar a sus víctimas y, llevar a cabo la trata y comercio sexual en dichas zonas turísticas, con la posibilidad de trasladarlas también al extranjero.

De igual manera, la importancia que tiene para detectar las células criminales y las zonas donde se encuentran las víctimas, lo que se llama perfil criminológico y el geográfico, así como también el psicogeográficos, tan útiles en la identificación del espacio en que existe la problemática, así como características de las víctimas y victimarios.

Así como también, se analiza la participación de diversos actores para se encuentran involucrados 
en el comercio sexual y, las estrategias de prevención que han implementado los países para lograr disminuir este abuso de poder contra, sobre todo, mujeres y niños.

\section{MIGRACIÓN Y MOVILIDAD POBLACIONAL}

Diversas son las formas y causas de la migración y movilidad poblacional en el mundo. A México, como ruta y puente para llegar a otros países, es muy común que llegue gente de otros países, ya sea se queden o sólo están de paso. Los motivos de este traslado son diversos, pero principalmente son de tipo económico.

La precariedad monetaria y otros factores, llevan a la gente a migrar hacia otros países, Estados o localidades, con la intención de mejorar su calidad de vida. Lo lamentable es que, en esa movilidad, existe gente que se aprovecha de ellos, y utiliza estas debilidades para cometer delitos de trata de personas, entre otros. Hay otros impulsos para la movilidad de estas personas, por ejemplo "el fracking, los cárteles de la droga, los feminicidios, las masacres y los asesinatos de autoridades locales, activistas de derechos humanos y ecologistas son las fuerzas necropolíticas detrás el desplazamiento de las poblaciones marginadas" (Estévez, 2018).

Esta movilidad involucra vulnerabilidad, sobre todo en mujeres y niños, que son más susceptibles a engaños por parte de los delincuentes, además de considerar los factores de riesgo que influyen para ser blanco fácil de las redes criminales. Por ejemplo, a México llegan migrantes de varias partes, pero sobre todo de centro américa, como lo menciona Cortés "Las mujeres migrantes centroamericanas son sujetos que realizan múltiples desplazamientos geográficos, geopolíticos y conceptuales o metafóricos en una suerte de movimiento multidimensional" (Cortés, 2018).

Estas mujeres lamentablemente, ya van etiquetadas desde la salida de sus países, ellas "portan en sus cuerpos físicos marcas sociológicas, culturales y políticas que los convierten en cuerpos generizados, racializados, etnizados, desterritorializados que cobran una gran relevancia en los regímenes de movilidad" (Cortés, 2018).

La movilidad interna en México, por ejemplo, en el Estado de Jalisco, se hizo un estudio (2008) en el Centro Preventivo y de Readaptación Femenil de Puente Grande, identificándose que “el 75\% de la muestra, migraron de otros Estados de la República Mexicana al lugar donde cometieron su delito, esto en correlación con otros factores y varios indicadores" (García, 2015).

Sin embargo, la mayor presencia de mujeres migrantes (2005-2010), fueron en Tabasco (53.8\%), Tlaxcala (53.7\%) e Hidalgo (53.4\%), con grupos de edades económicamente activas de 15 a 29 años (Romo, Téllez y López, 2013; García, 2015).

Existen muchas formas y utilización de medios para realizar la migración, al respecto, una de las 
teorías enfatiza que, los aportes que pueden derivarse del uso de la perspectiva de las redes sociales y del capital social para el entendimiento de reproducción de los circuitos migratorios. En este sentido, suele aceptarse que el estudio de la migración internacional pone de relieve el gran peso que tiene la conformación de redes sociales, especialmente a partir del trabajo pionero que hicieran Massey, Alarcón, González y Durand (1987), en el occidente de México (García, 2015).

De igual modo, se tiene a la migración interestatal en México (2002), que está integrada en su gran mayoría por migrantes urbano-urbano, esto es, que se desplazan de una ciudad a otra, fenómeno que forma parte de los nuevos flujos migratorios propios de una sociedad moderna. La migración municipal muestra un origen rural-urbano muy similar al de la estatal. Es muy probable que la desconcentración que están experimentando ciudades como Guadalajara, Monterrey, Puebla, Aguascalientes, y otras zonas metropolitanas, explique esta alta proporción de emigrantes municipales urbanos (Canales y Montiel, 2007; García, 2015).

Asimismo, se identificó la gran tendencia de la migración interna en México, lo cual modifica la dinámica delictiva, los grados de peligrosidad, planeación, instrumentos utilizados y los cambios en la autoría delictiva; esto conlleva a una mutación y evolución criminal de alto riesgo (García, 2015). Lo cual conlleva a incrementarse las áreas de oportunidad para que los grupos criminales, capten con mayor facilidad a sus víctimas, considerando sus necesidades, debilidades y vulnerabilidad.

\section{FACTORES DEL DESPLAZAMIENTO EN ZONAS TURÍSTICAS MEXICANAS}

Existen diversos factores para que las personas se desplacen, pero como ya dijimos anteriormente, es el económico el principal, y ya de ahí emanan todos los demás. En el caso de la migración de salvadoreños, las razones son multicausales, entre ellos, lo laboral, amenazas de grupos criminales, falta de oportunidades, bajos salarios y violencia, entre otros, prevaleciendo los de tipo económico (Ramos, 2013).

Podemos hablar de diversos países de América Latina y, en la generalidad son los mismos factores que motivan a una persona a moverse de su país. En este sentido, aunque la investigación se realizó en Cartagena, Colombia, los factores de riesgo se aplican igual en México, con variantes en base a los aspectos sobre todo culturales y participación de actores sociales, obviamente; esto nos ayuda a entender la problemática del comercio sexual en nuestro país. Dichos factores son (Bernal, et.al, 2013):

1. Factores Sociales

a. La emigración y el desplazamiento forzado: incide en las condiciones de vida de los Niños, Niñas y Adolescentes (NNA) y de sus familias. Como señala el estudio del ICBF y otros (2004) 
b. El aumento del turismo

c. Tolerancia al trabajo infantil

d. La lógica del consumo (sociedad reproduce continuamente los valores de la sociedad de consumo con la misma intensidad con que lo haría en los países más desarrollados.

e. Las deficiencias en el sistema educativo

f. Incremento en el consumo de estupefacientes

g. La existencia de redes (participación de diversos actores)

h. Inexistencia de redes de apoyo (para crianza de hijos)

2. Factores Económicos

a. Precariedad de las formas de sostenimiento

b. La familia como epicentro de vulnerabilidad: La vulnerabilidad no solo afecta

a las víctimas, es una realidad que atraviesa a las familias e, incluso, a algunos de los intermediarios de la explotación sexual (Grupo de Investigación en Derechos Humanos, 2011b).

\section{Factores Políticos}

4. Factores Culturales

En el caso de México, padres que venden a sus hijas para recibir algún estímulo económico, no solo se ve en pequeñas comunidades, sino en otros estratos sociales.

5. Otros Factores de Riesgo

a. La creciente utilización de TIC (internet y Redes Sociales)

b. El abuso sexual previo

c. La violencia intrafamiliar

De igual manera, otros autores refieren sobre el comercio sexual, considerar lo siguiente:

Causas y consecuencias sobre la gravedad de la trata de personas con fines de explotación sexual (Coca, Gaitán y Monras, 2016):

Factores económicos:

a) Falta de empleo.

b) Falta de alternativas laborales.

c) Pobreza.

d) El fracaso económico.

Factores sociales:

a) Madre soltera y cabezas de hogar.

b) la discriminación contra las mujeres.

c) Violencia intrafamiliar. 
d) Violencia sociopolítica.

e) Desastres naturales.

Factores culturales:

a) Bajo nivel educativo.

b) Prevalencia de objetivos económicos.

c) Valores sociales.

d) Desconocimiento de los reales alcances de la Trata.

Factores psicológicos:

a) Baja autoestima.

b) Antecedentes de maltratos.

c) Antecedentes de abuso sexual.

d) Antecedentes de prostitución y delincuenciales.

Como vemos con estos autores que exponen factores que impulsan la salida del ciudadano de diversos países, básicamente el común denominador es el económico, la violencia, el abuso, falta de políticas públicas eficientes para satisfacer las necesidades de sus pobladores, así como la explotación de que ya son objeto en el país de origen. Otros de los factores que participan en la construcción del comercio sexual, que tienen un trasfondo de género, son los roles y estereotipos que se generan desde la infancia, como refieren Celdrán y Quirante (2021: 6591), marcan un camino a seguir que diferencian desde el nacimiento a las niñas de los niños, condicionando sus decisiones, tanto personales, como profesionales y académicas a lo largo de toda su vida.

Existen otros desplazamientos en México, como casos y patrones de desplazamientos forzados causados por la violencia de los cárteles de la droga en un intento de desenmarañar las diferentes formas de movilidad humana y de distinguir las migraciones forzadas de las que no lo son. La proporción de personas que abandonan municipios violentos es entre 4 o 5 veces mayor que la de personas que abandonan municipios que no lo son y que se encuentran en las mismas condiciones socioeconómicas (Albuja, 2014).

Este desplazamiento forzado, en la última década se ha incrementado, obligando a los ciudadanos a abandonar sus hogares, aunque sean dueños, incluso, teniendo tierras que sembrar, por la inseguridad de que son objeto. Este desplazamiento los convierte aún más en seres vulnerables que, muchas veces no saben hacia dónde ir ni cuál será su destino, se encuentran perdidos, desplazados, ignorados y expuestos para la criminalidad, trata de personas, entre otros delitos, en su propio país, siendo el destino más atractivo, las zonas turísticas de México. 


\section{TURISMO SEXUAL Y REDES CRIMINALES}

Al estar expuesto, sea el migrante interno o externo en México o en otro país, por esa vulnerabilidad, es más factible sea captado para fines de comercio sexual, que es lo más común, en relación con otros delitos como la venta de drogas y otros más. Para entender el concepto de explotación sexual, se debe de analizar y empatizar con esta problemática; así, se define "como todo tipo de actividad en la que una persona usa el cuerpo de un niño, niña o adolescente para sacar ventaja o provecho de carácter sexual, basándose en una relación de poder" [Unicef, 2005]. Este “puede contener un componente comercial; y puede o no involucrar fuerza física, engaño o aprovechamiento, pero en todo caso implica convertir a los seres humanos en mercancías” (Bernal, et.al., 2013).

Como se ve, "el vínculo que la trata establece con el tema de la migración y la seguridad es notorio". Asimismo, "la trata/tráfico ilícito de migrantes, la confluencia de las intervenciones y discursos humanitarios y criminales hace que el tema de la 'víctima' se vuelva aún más complejo y delicado". Al respecto, Piscitelli y Lowenkron (Piscitelli y Lowenkron, 2015; Mansur, 2018), refiere "en sus reflexiones sobre los regímenes discursivos y legales sobre la trata de personas en Brasil y España, para acceder a algún tipo de reconocimiento, las personas 'víctimas de la trata' deben ser reconocidas como 'víctimas reales' por los Estados"; sin embargo, ya "en la práctica, los reconocidos como 'víctimas reales' son a menudo tratados como 'inmigrantes ilegales' a ser deportados o incluso criminalizados" (Piscitelli y Lowenkron, 2015; Mansur, 2018).

Como se identifica, la doble victimación en aquellos que han sido afectados por el tráfico ilegal de personas, es persistente, especialmente en mujeres y niños. Este problema va más allá de lo nacional, involucra una serie de acciones de corrupción, factores económicos, políticos, culturales, que forman redes criminales alrededor del mundo. De este modo, el tráfico de mujeres se ha esparcido a todos los países del mundo. En el caso de México, Hughes et al. (1999), citando a Hall (1998; Acharya, 2019), señalan que cada año cerca de 6000 a 8000 mujeres son traficadas desde estados como Guerrero, Chiapas, Oaxaca, Hidalgo, Puebla, Michoacán, Guanajuato, Campeche, Zacatecas, Colima, Veracruz y Quintana Roo hacia las grandes ciudades para la prostitución y un poco más de 5000 mujeres son traficadas desde México a Estados Unidos y Canadá. Según una estimación, de cada 10 mujeres de Chiapas en México, tres son traficadas fuera de la frontera y siete dentro del país (Acharya, 2019). Es decir, los traficantes prefieren traficar a las mujeres dentro de la misma frontera, porque según ellos se torna muy difícil llevar a una mujer a otros países a causa de la estricta vigilancia en la frontera y los aeropuertos (Acharya, 2019).

Identificando los puntos clave y principales en México, existen seis polos centrales para la explotación sexual, éstos son: Cancún, Acapulco, Ciudad de México, Ciudad Juárez, Tijuana y Monterrey (Acharya, 2019). En este sentido, Méndez (2006) apunta citando a Lidia Cacho, Monterrey es uno de los 
destinos principales para el tráfico de mujeres en México (Acharya, 2019).

Pero ¿qué tiene de particular estas ciudades? Entre otras, que son grandes metrópolis, 4 de ellas en fronteras, 3 hacia el norte y una el desembarco de Europa y los E.U. Otro de los factores comunes es que son zonas turísticas, atractivos para extranjeros generalmente, que, de antemano, se les ha ofrecido servicios sexuales, sea de niños, mujeres, hombres, sean nativos de la localidad a donde viajan o, captados des otros países por los grupos criminales.

Aquellos que participan en el comercio sexual, sea de manera voluntaria o no, nos preguntamos ¿por qué razón no se disuelven las células criminales que las explotan? ¿en qué está fallando la justicia? ¿por qué razón no importan estas personas víctimas de trata? ¿cuál será el destino de ellas?, pues estas mujeres traficadas son una población oculta, vulnerable y clandestina, es decir, no existe información sobre ésta, por lo cual es difícil contar con datos, como otro tipo de investigación (Acharya, 2019).

Sobre esta movilidad poblacional en zonas turísticas, para sobrevivir continúan siendo el usoobjeto sexual muy a pesar de estar en contra, pues no encontraron respuestas positivas al buscar oportunidades de vida, empleo, pobreza, vulnerabilidad, esto es el común denominador. De igual manera, obedece a ciertos fenómenos de la historia, como guerras, conquistas, movimientos sociales, cambios económicos, políticos.

Las víctimas, aquellos que son migrantes, p.e. de Centro América, por la precariedad en que viven, entre otros factores, se ven impulsados hacia los traficantes, esto asociado al uso de las nuevas tecnologías que facilitan la captación de personas para traficación. Así, la movilidad con todo y los motivos de ésta, es el principal punto vulnerable de las víctimas, que a su vez, es un factor de riesgo y una oportunidad idónea que aprovechan los traficantes para captar víctimas, su transporte, el traslado, la acogida o la recepción de personas utilizando "amenazas o al uso de la fuerza u otras formas de coacción, al rapto, al fraude, al engaño, al abuso de poder o de una situación de vulnerabilidad o a la concesión o recepción de pagos, beneficios para obtener el consentimiento de una persona que tenga autoridad sobre otra, con fines de explotación“ (Protocolo de las Naciones Unidas, 2000, para prevenir y sancionar la trata de personas, especialmente de mujeres y niños que complementa la Convención de las Naciones Unidas contra la Delincuencia Organizada Transnacional, define en el artículo $3^{\circ}$, la trata de personas) (United Nations General Assembly, 2000; Organización de las Naciones Unidas, 2000; Acharya, 2019).

El tráfico de mujeres es un fenómeno que tiene raíces en la historia y que estuvo ligado desde sus orígenes a las guerras, a la esclavitud y a la objetivación sexual de las mujeres. Durante la época de los descubrimientos geográficos, las mujeres, principalmente las nativas africanas, eran traficadas como esclavas con un triple propósito: trabajar como mano de obra gratuita, la reproducción de esclavos, o sea, la producción de más mano de obra gratuita, y servir de objeto sexual. Este último propósito estaba 
siempre presente, aunque el objetivo principal fuera cualquiera de los otros dos y podía darse dentro del mercado matrimonial o con otras figuras como concubina, o simplemente mujer libre a disposición del patrón (Chiarotti, 2002; Acharya, 2019).

La trata de personas es un problema con historia y, que continúa, se refuerza utilizando todos los medios posibles para lograrlo. Durante todo el siglo XX, después de cada guerra mundial, el tráfico de mujeres continuó, siendo también víctimas del mismo las de Europa, que, huyendo del hambre y el horror de la guerra, eran presa fácil de los traficantes. Esto llevó a denominar a la actividad como trata de blancas, misma que luego se transformó en trata o tráfico de mujeres (Acharya, 2019).

El fenómeno del tráfico ha tenido un gran desarrollo sobre todo en las últimas tres décadas. A principios de los años 80, miles de mujeres extranjeras llegaban a Europa y América en búsqueda de empleo y mejores condiciones de vida. Pero debido a sus condiciones de ser ilegales, pobres y vulnerables, pronto cayeron en organizaciones criminales dedicadas a la industria del sexo (Acharya, 2019).

En la actualidad, este fenómeno se ha convertido en un problema global que involucra una compleja matriz que va desde el origen, el tránsito, hasta llevar al destino, incluso tiene que ver con las relaciones internacionales y la economía de los países, además de que existe una complicada red de personas que están involucradas (Acharya, 2019).

Así, tenemos un problema a nivel mundial, cuyos actores son diversos, incluyendo la política, empresariales, y quienes ejercen la justicia, por ello, es mucho más difícil lograr la captura y enjuiciamiento de los criminales, quienes ostentan el poder y recurso económico a grandes escalas. Entre estos actores sociales participantes en la criminalidad, se identifican ciertos perfiles específicos que se van generando desde su formación de estos individuos desde la niñez hasta la adultez, no sorprendiendo que lleguen a cambiar de bando, es decir, de estar del lado de la justicia, trabajando para el Sistema jurídico y penal, e incluso en el militar, pasen a pertenecer a una organización criminal, como en el caso que nos explica Tiffon (2021: 5531), en el que explica los rasgos de personalidad de base en un caso de asesinato que se perpetró por un sujeto que desarrollaba actividades profesionales en el ámbito militar de élite, y las repercusiones jurídico-legales que le supuso en el delito de asesinato a un civil. Como vemos, ese cambio tan factible y, fácil de, tal vez, una persona intachable en su carrera militar a la vida criminal, y que se da en cualquier país y, hablando de México es tan común que, llegan a escoger estas personas expertas en armas y otras estrategias y habilidades, un cambio de vida o, se las ofrecen con mejores condiciones, siendo reclutados para fines delictivos, como lo es de una organización criminal dedicada al tráfico de personas y comercio sexual.

En el caso de América Latina, el tráfico de mujeres se remonta a la época de la Conquista cuando los españoles. En la actualidad, en América Latina, por sus características, puede dividirse en dos: el 
tráfico interno, que se refiere a la demanda existente en el propio país, y el tráfico externo, que incluye una demanda en el mercado internacional (Molina, 1995; Acharya, 2019).

El punto de partida generalmente aceptado es que los migrantes, bajo ciertas condiciones propias de su entorno social como precariedad económica, violencia familiar, desempleo, discriminación sociocultural, por lo que se ven forzados a buscar los servicios de los traficantes, constituyéndose estas condiciones en factores esenciales para explicar el fenómeno (Salt y Stein, 1997; Acharya, 2019). Precisamente es de esta vulnerabilidad de la que se habla con respecto a las víctimas, llegan a un punto en que no encuentran una salida a sus problemas, generalmente económicos, y, por ende, buscan apoyo donde sea y como sea; lo más notorio es que no solo se involucran con los criminales de manera individual, sino que, incluyen a sus familiares e incluso a amigos.

De acuerdo con los autores Skrobanek et al. (1997)esta actividad no es un fenómeno nuevo, quizás lo nuevo es su sofisticación global y el uso de las tecnologías modernas de comunicación que utilizan, lo cual ha incrementado el número de mujeres y niños traficados, (Acharya, 2019) de acuerdo con esto, como ya habíamos afirmado anteriormente, los integrantes de las redes criminales se van modernizando, utilizando las nuevas tecnologías para lograr sus fines con mayor facilidad, traficando con personas a cualquier parte del mundo, ya que han formado alianzas con organizaciones delictivas de otros países.

Ante este planteamiento, la migración y el tráfico de personas se pueden analizar a partir de la movilidad de la gente y con frecuencia se hacen distinciones vinculadas con actividades del mercado laboral, como el servicio doméstico o el trabajo agrícola, por ejemplo (Acharya, 2019).

Al interior de la República Mexicana, se señala que cada día, por medio de cinco rutas, llegan a Tijuana mujeres y niñas de entre 3 a 65 años de edad para ser forzadas a prostituirse. Según este reporte, la mayoría de estas mujeres provienen de estados como Oaxaca, Sinaloa, Chiapas, Jalisco, Michoacán, Nayarit, Puebla, Guerrero, Durango, Distrito Federal, Veracruz, Estado de México y Baja California (Alto Comisionado de las Naciones Unidas para los Refugiados (ACNUR), 2008; Acharya, 2019).

Asimismo, en la Zona Metropolitana de Monterrey las mujeres víctimas del tráfico proceden en su mayoría de lugares pobres, donde existe la miseria y la falta de oportunidades. Estas situaciones son precisamente de las que se aprovechan los proxenetas para engañar a sus víctimas. En general, las mujeres traficadas que se encuentran en esta zona urbana son de diferentes estados del país, pero existe una alta concentración de mujeres de los estados del norte y centro. Principalmente estas mujeres provienen de estados como: San Luis Potosí, Zacatecas, Veracruz y Coahuila (Acharya, 2019).

Sobre las dinámicas del mercado ilegal que opera en los viajes y el turismo que busca menores de edad para el mercado del sexo, sumado al consumo de drogas ilegales, tenemos un problema a nivel mundial. 
Los viajes y el turismo asociados a la escnna en Colombia, sumado a la pornografía infantil, son realidades que se han venido dando en aumento a lo largo del tiempo. Desde tiempos remotos, la sexualidad ha desempeñado un papel en el cual las dinámicas de la guerra, las conquistas, la política, la economía y la religión han estado presentes, y en este sentido, el modelo patriarcal hegemónico no ha sido tendiente a hacer prevalecer, ni proteger los derechos de los no favorecidos, entre ellos, por lo general, el de las mujeres y los menores de edad. Sin embargo, pese a la "normalización" del uso de la fuerza sobre mujeres y menores de edad enmarcado por la costumbre, estos no tenían voz, y poco a poco con el paso de la historia las luchas y reivindicaciones sociales conllevaron que algunas sociedades, entre ellas las francesas e inglesas, repensaran los derechos en igualdad de condiciones para todos y se protegiera a los más débiles (Humbarita, 2015; Sánchez, 2019).

En nuestra sociedad, ha coexistido una vulneración hacia los derechos de niños, niñas y adolescentes, en que la explotación del ser humano ha supuesto que se asuma en muchos casos la "normalización" de la "prostitución" a temprana edad con menores de edad, cuando la prostitución corresponde a un mundo adulto, en que la autonomía y voluntariedad no son elementos a tomar cuando se involucran menores de edad para actividades sexuales o eróticas (Sánchez, 2019).

La explotación del ser humano, en cualquiera de sus variantes, se ha normalizado, como el comercio sexual por dinero, como cualquier otro negocio, donde en México, en sus zonas turísticas como Cancún, prevalecen las familias dedicadas a este tipo de conducta, viéndolo y justificándose como "actividad normal, como hacer un masaje".

Otro aspecto importante es, el de la "permisividad" por parte del Estado para ejercer el comercio sexual, sin aplicar la ley.

En esa movilidad entre países para la compra-venta de sexo, mediante viajes de "placer", es una práctica muy común en zonas turísticas, sobretodo.

La problemática en México, así como en otros países, es que el pretexto de las crisis económicas, la práctica "normal" de la venta del sexo, parte no sólo de las Redes Criminales, sino de las propias familias que ven este negocio muy fructífero y sin riesgos, enseñando a los hijos a ejercerlo como cualquier otro trabajo, es decir, favorece a la movilidad de la compra-venta de sexo; este nuevo nivel de prácticas de comercio sexual, por parte de "familias", a veces completas; e incluso en la movilidad externa e interna, ya muchos de los niños y jóvenes, saben perfectamente en lo que trabajarán, son conscientes de lo que tienen qué hacer y para qué los captan las redes criminales ¿y contra esto, qué podemos hacer, cómo protegerlos?. Desde luego, es una nueva vertiente, un nuevo paradigma, en el que hay que trabajar, investigar.

Es entonces el turismo sexual un problema no solo nacional sino mundial que requiere políticas 
públicas contundentes en la protección de los derechos fundamentales de los menores. Por ende, las víctimas de delitos sexuales son menores de edad (niños, niñas y adolescentes) en los que el comercio sexual, la pornografía infantil y el turismo con fines sexuales no se denuncia por temor, amenazas, y en muchos casos, peligra su vida. Por ello, el trabajo y el deber de las autoridades por proteger sus derechos se hace una tarea muy compleja, pues no es suficiente la fuerza pública y personal capacitado para salvaguardar sus derechos (Sánchez, 2019).

Respecto a las políticas públicas en el turismo sexual y su movilidad, deberán de sustentarse y apegarse a la realidad social existente, para que sean eficaces, pensar en los nuevos mecanismos, modus operandi, cambios de formas de pensar, visiones modernas del ejercicio del comercio sexual, a nivel interno y externo de los países.

Considerar que, la migración criminal en zonas turísticas mexicanas, es para ejercer cualesquiera de los delitos, generalmente de manera mixta, entre ellos práctica del turismo sexual, el cual está en profundo cambio, en relación a los valores-antivalores de la misma sociedad cada vez más resquebrajada y vulnerable.

De la misma manera, "si hay algo que caracteriza al turismo sexual es precisamente su dimensión internacional, porque recibe turistas de cualquier país, y utilizando sólo los recursos materiales, sino también los humanos, y los lleva hasta extremos absolutamente degradantes para aquellas personas que caen en sus redes y no pueden salir" (Martínez, 2006; Ruíz, 2017).

A nivel mundial tenemos que, según las estimaciones de EUROPOL, la trata de seres humanos constituye la segunda fuente de ingresos ilícitos de la delincuencia por detrás del tráfico de drogas, moviendo anualmente unos 32.000 millones de dólares. La ONU indica que la trata de personas con fines de explotación sexual mueve en el mundo entre 5.000 y 7.000 millones de dólares al año, lo que representa aproximadamente el $85 \%$ de las ganancias totales por el delito de la trata de personas cuya cantidad asciende hasta los 10.000 millones de dólares anuales (Ruíz, 2017).

Sobre las redes criminales, son complejas, no sólo por su tamaño, sino por su capacidad de penetrar y manipular múltiples facetas de la sociedad. Estas complejas redes transnacionales implican múltiples entidades criminales (sumando alianzas entre grupos delictivos), participando igual negocios, empresas, oficiales corruptos, políticos (Salcedo y Garay, 2016).

Para analizar esta problemática, es imprescindible tener una visión multidisciplinaria y multifactorial. En este sentido, el macro análisis sobre la configuración de una red en conjunto, se hace para entender el potencial de transformación de una red y sus interacciones con otras redes. Así, dicha macro perspectiva, informa acerca de la resiliencia de la red y sus formas de organización. En México existen, al menos tres estructuras que, convergen en una misma red criminal: una es una estructura 
coercitiva, la otra política y finalmente la financiera, que son muy importantes en la operación de la red criminal (Salcedo y Garay, 2016).

Para realizar un análisis efectivo sobre turismo sexual y redes criminales, es indispensable utilizar “el aspecto Geográfico Criminal”, el cual "podría definirse como un conjunto de factores que permiten un análisis espacial o demográfico de las zonas donde se cometen delitos y su vinculación íntima con el delincuente, aportándole a los investigadores datos geoespaciales", en este caso sería "como ser vías de escape, accesos a asentamientos precarios, terrenos o zonas destinadas al descarte de elementos propios del ilícito, zonas con mayor presencia de personal policial o cámaras de seguridad viales, entre otros”. Se determina que "todos estos datos aislados, pero analizados en su conjunto permiten la elaboración de las diferentes hipótesis criminales, descartando las menos probables y centralizando todos los estudios y tareas en las que posean mayor probabilidad de certeza", así, de este modo se originarían "las primeras medidas judiciales de prevención, cautelares o de investigación tendientes todas ellas al esclarecimiento del hecho pasado investigado por el magistrado correspondiente" (Esteller, 2014).

Posteriormente, “estos datos geoespaciales incorporados en un Sistema de Información Geográfica ("SIG") ofrecen la capacidad de brindarle al usuario la posibilidad de determinar zonas comunes de actuación, de mayor índice delictual, prever sus movimientos y bloquear las vías de escape posibles conformando cerrojos vehiculares o personales" (Esteller, 2014).

Y al mismo tiempo, este "aspecto geográfico permite la demarcación de zonas críticas o peligrosas, poco o densamente pobladas, ofreciéndole a las autoridades correspondientes de datos sensibles para favorecer la toma de decisiones", tomando un ejemplo, siendo "información socio-cultural, cantidad de población estable, cantidad de personas circulantes o en tránsito en una zona determinada, establecimientos educacionales, sanitarios, policiales o de seguridad, estado de los caminos, cursos de agua con sus elevaciones máximas para la determinación de crecidas, entre otros”. Una vez lo anterior, “estos datos informáticos incorporados en las diferentes plataformas SIG facilitan la distribución de recursos aprovechándolos al máximo sabiendo donde ubicarlos para abarcar la mayor zona posible y su equitativa aplicación poblacional" (Esteller, 2014).

De vital importancia es el Perfil Geográfico Criminal, ya que es "una técnica de análisis que complementa a toda la investigación criminal, basándose en el uso de herramientas SIG los cuales permiten la incorporación de datos temáticos propios de cada usuario aplicados a delitos específicos”, los cuales sirven de una "gran variedad de usos, aplicaciones personalizadas y análisis particulares según la temática y la zona de estudio" (Esteller, 2014).

Sobre el perfil geográfico, existen "nuevas investigaciones y recientes propuestas, a nivel internacional, es el desarrollo de un Geographic Profiling o Perfil Geográfico en español, conocido como 
Retrato Geográfico en otros países. Es el producto de la intersección de tres ciencias: la geografía, la psicología y la criminología con el objetivo de localizar el área más probable donde puede encontrarse al agresor: la residencia, lugar de trabajo u otro conocido como punto de anclaje, en función al análisis geoespacial” (Reyes y Estrella, 2019).

Involucra el espacio, lo propio del sujeto, teorías de oportunidad, el modus operandi, entre otros. Así, este "Perfil Geográfico tiene sus bases en la CA, debido a que estudia la distribución en el espacio y tiempo de los hechos delincuenciales a tal punto de identificar "dónde y cuándo ocurren los delitos, bajo qué influencias ambientales se producen, y cómo estos conocimientos pueden ser útiles para predecir, controlar e incluso prevenir eventos delictivos" (Vozmediano y San Juan, 2010, 43], como también para detener a los criminales futuros" (Reyes y Estrella, 2019).

Respecto a las "teorías que sustentan a la criminología ambiental tratan de explicar cómo el entorno tiene un efecto sobre el comportamiento delictivo y cómo los patrones describen las actividades de las víctimas y delincuentes", donde se describe "la Tabla 2 (Martínez, 2016) (Teorías de oportunidad del delito aplicado a la criminología ambiental): Teoría de las Actividades Rutinarias, Cohen y Felson (1979); Teoría de la Elección Racional, Willson y Herrnstein (1985), Clarke y Cornish (1986); Teoría de las Ventanas Rotas, Wilson y Kelling, (1982), Skogan (1990), Kelling y Coles (1996); Teoría del Patrón Delictivo, Brantingham y Brantingham (1991) (Reyes y Estrella, 2019).

La ciencia va avanzando, utilizando de igual manera las nuevas tecnologías para realizar el Perfil Geográfico, ya que se "puede aplicar una herramienta fuerte como la es el análisis espacial, que se centra en el estudio del comportamiento de los diferentes elementos en el espacio bajo ciertas condiciones", esto con la finalidad de: "elaborar teorías generales o modelos espaciales, comprender la naturaleza del fenómeno, identificar relaciones entre los individuos y el espacio, analizar una problemática social, determinar cuáles son los agentes transformadores, y otros” (Reyes y Estrella, 2019).

Por lo tanto, "el proceso de análisis espacial en la aplicación de las teorías de oportunidades delictivas inicia con la sociedad en el sentido amplio de las actividades rutinarias, que permite llegar al área local para analizar el patrón delictivo y por último enfocarse con el individuo en función a la elección racional" (Reyes y Estrella, 2019).

Así que, el "Perfil Geográfico Criminal permitirá apoyar el proceso de toma de decisiones con el fin de generar un elemento clave y complementario para la Policía Nacional, servicios de inteligencia y con ello lograr aumentar las medidas de localización de agresores para reducir el efecto de inseguridad, y acercándose cada vez más a los objetivos nacionales e internacionales propuestos” (Reyes y Estrella, 2019).

Para un perfil geográfico, es necesario un perfil criminal, en este sentido, "a partir de 1970, el 
perfil criminal es establecido como una técnica de investigación utilizada para resolver casos difíciles y se conforma la unidad de análisis de la conducta del FBI” (Maldonado, 2016).

Tipos de perfiles criminales (Maldonado, 2016)

1. Perfiles de agresores conocidos-método deductivo

2. Perfiles de agresores desconocidos- método inductivo

3. Perfil geográfico

Los perfiles van cambiando, conforme evolucionan las conductas, los modus operandi, la misma sociedad. En este sentido, no quedan fuera las mujeres, que en algún momento de sus vidas han sido víctimas de abusos, especialmente de delitos sexuales, al convertirse en victimarias, suelen repetir la conducta del que fueron objeto, sea en su niñez o adolescencia, además de ir más allá, cometiendo delitos de homicidio, pudiendo llegar a convertirse en asesinas seriales, sea de un tipo específico de víctimas y/o de la propia familia (García, 2011). Al respecto, es muy importante tener en cuenta, la historia de vida de estas mujeres, quienes en un 90\% fueron víctimas, y llegaron en algún momento de sus vidas, a ejercer la prostitución, sea forzada o no.

Los cambios en las conductas de las mujeres y el papel que representa en la actualidad, cuando de víctima se convierte en victimaria, por ejemplo, sus víctimas son preferentemente personas vulnerables como niños, ancianos o personas enfermas que necesitan cuidados o bien personas confiadas en exceso por la existencia de una relación sentimental, familiar o de amistad, de este modo se verifica que en la mayoría de los casos conocen a las víctimas (Egger, 1999; Hickey, 1997; Mariño, 2020).

A parte del perfil de las víctimas y de los delincuentes, en esta movilidad criminal se establece que, el perfil geográfico está íntimamente relacionado con las características físicas del lugar donde es cometido el crimen, el cual podría llamarse "perfil de la escena del crimen" (Rodríguez, 2011; Vaca y Aguilar, 2012; Mariño, 2020).

La siguiente técnica puede ayudar a identificar las células de las Redes Criminales, su movilidad hacia zonas turísticas al interior del país e, inclusive al exterior, donde trabajan para el tráfico de personas, y su modus operandi:

Rossmo y Summers (2015; Mariño, 2020) ha destacado la importancia de utilizar programas informáticos para estudiar las características espaciales de los delitos seriales, empleando una fórmula matemática denominada: criminal geographic target (CGT), donde tras introducir ciertos datos como: punto exacto de las escenas de los crímenes, datos de los testigos que presenciaron los hechos o que puedan aportar algo relevante para la investigación y el estudio del análisis demográfico de los lugares donde había secuestrado a las víctimas, o localizado los cadáveres, el área afectada por dicho crimen, junto con unos cálculos matemáticos proporcionaba tres importantes datos: 
- La zona de "buffer zone" o zona de confort o seguridad.

- Patrones de caza o rutas escogidas por el delincuente.

- La posible área de ubicación de su domicilio o base de operaciones.

Muestra que es posible identificar patrones de comportamiento geográfico en criminales y usar esa información para crear herramientas que faciliten la labor policial definiendo áreas posibles de su punto de anclaje. Esta técnica está pensada para sucesos seriales, debe darse repetición y acumulación de datos (Mariño, 2020).

Rossmo y Summers (2015; Mariño, 2020) estableció una tipología, al igual que Canter (2007; Mariño, 2020), en base a la movilidad del delincuente teniendo en cuenta la metodología que éste utiliza en la búsqueda de su víctima:

Cazador (Hunter): busca a sus víctimas en los alrededores de donde vive. En la clasificación de Canter serían los merodeadores.

- Cazador furtivo (Poacher): busca una víctima teniendo como base de operaciones un lugar diferente de su casa, o bien se traslada a otra ciudad para seleccionarla. En la clasificación de Canter equivaldría a los viajeros.

- Pescador (Troller): el agresor actúa en su zona de actividad rutinaria aprovechando una oportunidad que se le brinda.

- Trampero (Trapper): el agresor asume una posición o crea una situación por medio del engaño, que le permite encontrar víctimas en un lugar que él domina.

Asimismo, Rossmo y Summers (2015; Mariño, 2020) creó en la Universidad de Vancouver el programa Rigel, primer software específico para la realización de perfiles geográficos. Se trata del principal sistema utilizado para crear geoperfiles y está basado en el algoritmo CGT, donde el modelo analiza los patrones de las localizaciones relacionadas con una investigación criminal y genera una superficie de riesgo que indica la probable residencia del autor, por medio de un mapa de color de dos o tres dimensiones llamados jeopardies. La información puede incluir escenas del crimen, sospechosos, detalles del caso y del investigador.

Así, en la movilidad delincuencial, es necesario realizar perfiles, en específico, geográficos; pero no solo eso, sino que, como Maurice Godwin (EE.UU) (Godwin, 2006; Mariño, 2020) va más allá, él acuñó la expresión “elaboración de perfiles psicogeográficos”, que se basan en la recopilación y el análisis crítico de más de cien mil datos y doscientos actos concretos en escenarios del crimen, que pueden ser utilizados para trazar un perfil del autor de los hechos y utilizando el programa de software para elaborar perfiles geográficos que desarrolló, llamado "Predator", puede predecir con exactitud en qué lugar actuará un asesino, así como su zona de residencia. Su fundamento se sustenta en la inclusión de diversos 
parámetros, como el estudio de las distancias del delito, el análisis demográfico, la psicología ambiental, el análisis del paisaje, los sistemas de información geográfica, la delincuencia residual, etc. Las características distintivas con respecto a los otros programas, es que no resalta que el área de la delincuencia sea sólo circular, sino también angular. El software usa una gama de colores para mostrar en el mapa las dispersiones/ concentraciones de sucesos y la zona probable de anclaje.

En la actualidad existen muchos programas, sistemas y técnicas para realizar un perfil geográfico criminal, que apoye en la identificación de sujetos involucrados den redes criminales, y que utilizan la movilidad de sus víctimas para ejercer el comercio sexual. Del mismo modo, estos mismos criminales, se mueven de manera interna y externa al interior y exterior del país, con la finalidad de detectar y capturar a sus víctimas.

\section{PREVENCIÓN DEL COMERCIO SEXUAL}

Para la prevención del comercio sexual, no es nuevo la utilización de estrategias, creación de programas, leyes y demás mecanismos para la protección de la víctima y erradicación del problema.

En cuestión de políticas de prevención, la Organización Mundial del Turismo (OMT) (2001) y la Asociación Internacional de Hoteles y Restaurantes en Europa, apoyaron ésa iniciativa. La OMT en particular, consideró éste asunto en su declaración sobre la prevención del turismo sexual organizado de 1995. Este fue el primer documento foral adoptado por miembros de la OMT que trata directamente sobre la explotación sexual infantil (Aguilar, 2005).

La importancia de la participación de todos los sectores de la sociedad de un país y entre países, es muy importante, para prevenir este tipo de daño social a las víctimas.

Otro de los avances en esta problemática, la tenemos en 1996 con el Primer Congreso Mundial en Estocolmo Suecia que reunió a más de 1300 delegados de 122 países que adoptaron la Declaración y Programa de Acción elaborados a modo de directrices para la coordinación y la cooperación a escala local, nacional e internacional, la protección de los niños frente a esa explotación, la recuperación y reintegración de las víctimas y la participación de los niños en la lucha (Aguilar, 2005).

Consecuentemente, tras los congresos internacionales, la OMT (Organización Mundial del Turismo 2001) creó el Grupo de Protección Infantil contra la Explotación Sexual en el Turismo integrado por representantes de gobiernos, organismos intergubernamentales, organizaciones no gubernamentales, asociaciones del sector turismo, ECPAT y medios de comunicación. La misión muy importante consiste en prevenir, descubrir, aislar y erradicar la ESCI en el Turismo (Aguilar, 2005).

De igual manera, participó en mejoras en el tráfico de personas, la Unión Europea, que preparó y puso en práctica diversos programas encaminados a combatir la ESCI en el turismo: Un ejemplo de esas 
medidas, lo constituyen los módulos formativos que imparte la Fundación Por Nuestros Niños de Puerto Vallarta y el Centro Universitario de la Costa desde principios del 2003 (Aguilar, 2005).

Y respecto a nuestro México, el Centro de investigaciones y Estudios Superiores de Antropología Social realizó el primero durante dos años en seis ciudades del país que tenían antecedentes sobre la existencia de la ESCI, arrojando el dato de que, en México, más de 16,000 niños, niñas y adolescentes han sido víctimas de la prostitución infantil (Azaola, 2000; Aguilar, 2005).

Como hemos notado aquí, y analizado, la problemática de la migración criminal a zonas turísticas mexicanas, no es un asunto exclusivo de esas zonas, ni de este país, sino de un conjunto de actores nacionales e internacionales que apoyen a controlar el tráfico de personas, la compra y venta del sexo; y la creación de mecanismos efectivos que incluyan resultados positivos, sin quedar solamente en una reunión de buena voluntad y en un papel justificativo, para ello, unas adecuadas y aplicables políticas públicas, partiendo de la realidad social sobre, por una parte, empresas nacionales e internaciones criminales y, por la otra, la prevención para las posibles víctimas, que sean realmente eficaces, solamente así, se podrá tener logros en este problema. 


\section{REFERENCIAS}

Acharya, Kumar A. (2019), Tráfico de mujeres hacia la Zona Metropolitana de Monterrey: una perspectiva analítica, Espacios Públicos, vol. 12, núm. 24, abril, 2009, pp. 146-160. En: http://www.redalyc.org/articulo.oa? $\mathrm{id}=67611167009$

Aguilar González, L. A. (2005), La Explotación Sexual Comercial Infantil (ESCI) en el Turismo. Análisis del turismo sexual internacional que afecta a la niñez. Pasos, Revista de Turismo y Patrimonio Cultural, Vol. $3 \mathrm{~N}^{\circ} 1$ págs. 207-210. www.pasosonline.org.

Albuja, S. (2014), Violencia criminal y desplazamiento en México, RM 45. En: https://rua.ua.es/dspace/bitstream/10045/36448/1/RMF_45_09b.pdf Alto Comisionado de las Naciones Unidas para los Refugiados (ACNUR) (2008), Las rutas del tráfico de Mujeres, en www.acnur.org/paginas/?id_pag=7068, consultado el 6 de agosto, 2008.

Azaola E., (2000) "Infancia robada “. Centro de Investigaciones y estudios Superiores en Antropología Social.

Bernal-Camargo, D. R., Varón-Mejía, A., Becerra-Barbosa, A., Chaib-De Mares, K., Seco-Martín, E. \& Archila-Delgado, L. (2013). Explotación sexual de niños, niñas y adolescentes: modelo de intervención. Revista Latinoamericana de Ciencias Sociales, Niñez y Juventud, 11 (2), pp. 617-632.

Canales A. I \& Montiel Armas I., (16 de abril de 2007).Taller Nacional sobre Migración interna y desarrollo en México: diagnóstico, perspectivas y políticas. De la Migración Interna a la Internacional. En Búsqueda del Eslabón Perdido. Pág. 35.

Canter, D. (2007). Maping Murder: The Secrets of Geographical Profiling. UK: Editorial Virgin Books. Celdrán Fernández, E.M.y Quirante Martínez, O. (2021), La transmisión de estereotipos y roles de género a través del discurso de la maestra/de educación infantil. Vol. 2 No. 4 (2021): South Florida Journal of Development, Miami, p. 6585-6607 v. 2, n. 5, oct./dec. 2021.

Coca Carvajal, A. R.; Gaitán Cocuy, C. C.; Monras Torres, J. (2016), La explotación sexual como una forma de trata de personas, (Inf. Tesis Posg.) Universidad Santo Tomás, Facultad De Derecho, Especialización En Derecho Administrativo, Villavicencio. En: https://repository.usta.edu.co/bitstream/handle/11634/12628/2016adrianacoca.pdf?sequence=1\&isAllow $\mathrm{ed}=\mathrm{y}$

Cortés, Almudena (2018) Violencia de género y frontera: migrantes centroamericanas en México hacia los EEUU, European Review of Latin American and Caribbean Studies / Revista Europea de Estudios Latinoamericanos y del Caribe, No. 105 (January-June 2018), pp. 39-60. Published by: Centrum voor Studie en Documentatie van Latijns Amerika (CEDLA). En: URL: https://www.jstor.org/stable/10.2307/26525008

Chiarotti, S. (2002), “Trata de mujeres: conexiones y desconexiones entre género, migración y derecho humanos", en Conferen cia Hemisférica sobre Migración Internacional: derechos humanos y trata de personas en las américas, noviembre, CEPAL, Santiago de Chile.

Egger, S. (1999). Psicópatas y Asesinos en Serie. Cuarta Reunión internacional de biología y sociología de la violencia. Centro Reina Sofía para el Estudio de la Violencia. Valencia, España. 
Esteller, G. (Dic 2013-Feb 2014), “Mapa del delito o geografía criminal”. En SKOPEIN, la justicia en manos de la ciencia. Revista Criminalística, Año 1, Número 2. Reedición número 1 (Enero 2014). www.skopein.org - info@skopein.org

Estévez, A.. (2018). El dispositivo necropolítico de producción y administración de la migración forzada en la frontera Estados Unidos-México. Estudios fronterizos, 19, e010. Epub 22 de mayo de 2018.https://doi.org/10.21670/ref.1810010.

García Álvarez, M. F. (2015). Migración en la criminalidad / Migration in crime. RIDE Revista Iberoamericana Para La Investigación Y El Desarrollo Educativo, 6(11), 787 - 795. Recuperado a partir de http://ride.org.mx/index.php/RIDE/article/view/128

García Álvarez., M.F. (2011) Análisis de las Causas de la Conducta Delictiva de la Mujer en Jalisco, desde la Perspectiva Socio-Jurídica y Criminológica, (Tesis Doctorado) Cuernavaca, Morelos, México.

Godwin, M., Rosen, F.S. (2006): El rastreador. El perfil psicogeográfico en la investigación de crímenes en serie. Ed.Alba: Barcelona.

Hall, J.N. (1988), "The community-based Drug Epidemiology Network", Health directorate of the Commission of the European Communities, Brussels en Oyen Else (eds.), Comparative methodology: Theory and practice in International Social Research, sage publication, California, 1990.

Hickey, E.W. (1997). Serial murderers and their victims. Belmont, CA: Wadsworth Publishing Company. Humbarita, J. I. (2015). Derecho constitucional hispanoamericano frente a la realidad institucional: manifiesta divergencia. IUSTA, 2(43), 91-118. DOI: https://doi.org/10.15332/s1900-0448.2015.0043.03 Maldonado Gutiérrez, W. (2016). Consideraciones acerca del concepto "perfil criminal”. Duazary, 8(1), 115-119. https://doi.org/10.21676/2389783X.1680

Mansur Dias, G. « Trata de personas, tráfico de migrantes y la gobernabilidad de la migración a través del crimen », Etnográfica [Online], vol. 21 (3)|2017, Online desde 24 março 2018, consultado em 01 agosto 2021. URL : http://journals.openedition.org/etnografica/5026;DOI: https://doi.org/10.4000/etnografica.5026

Mariño Pérez, M. (2020), Análisis del Perfil Geográfico de Juana Barraza Samperio "La Mataviejitas", Revista de Criminología, Psicología y Ley. Vol. 3. En: https://educacion.usal.es/wpcontent/uploads/sites/46/2020/02/COMPLETADEF.pdf

Martínez Rolg, A. (2016). Criminología Ambiental y SIG, una aplicación práctica en Castellón de la Plana. Tesis de grado. Universitat JAUME I. Disponible en: http://repositori.uji.es/xmlui/bitstream/handle/10234/161313/TFG_Marti'nez\%20Roig_Angel.pdf?seque nce $=1$ [Consultado: 01-04-2019].

Martínez, V., (2006), Ocio y turismo en la sociedad actual. Los viajes, el tiempo libre y el entretenimiento en el mundo globalizado, Madrid: McGraw Hill.

Méndez, R. (2006), “Cancún capital del tráfico de mujeres”, en Revista Contralínea, Año 1, Núm.4, Quintana Roo, México.

Molina, F. P. (1995), "El tráfico de mujeres en América Latina”, en www.alainet.org., consultado el 13 de septiembre, 2004. 
Organización de las Naciones Unidas, (2000), "Convención de las Naciones Unidas Contra la Delincuencia Organizada Transnacional y sus Protocolos”, Viena: Oficina de las Naciones Unidas contra la droga $\mathrm{y}$ el delito. Disponible on line: https://www.unodc.org/documents/treaties/UNTOC/Publications/TOC\%20Convention/TOCebook-s.pdf Organización Mundial del Turismo 2001 "La Protección de los Niños contra la Explotación Sexual en el Turismo “.

Piscitelli, A., y Lowenkron, L. (2015), "Categorias em movimento: a gestão de vítimas do tráfico de pessoas na Espanha e no Brasil”, Ciência e Cultura, 67 (2): 35-39.

Ramos, E. (2013), Migración Forzada y Violencia Criminal: una aproximación teórico-práctica en el contexto actual, (Investigación) Universidad Tecnológica de El Salvador, Colección Investigaciones v. 37, San Salvador.

Reyes-Yunga, D.; Estrella, C. (2019). El perfil geográfico criminal una nueva propuesta para la investigación geoespacial de delitos. Revista GEOESPACIAL, 16/2: 16-32.

Rodríguez, J. (2011). La perfilación criminal como técnica forense en la investigación del homicidio. Revista de la Escuela de Medicina Legal, febrero de 2011, 4- 13.

Romo Viramontes, R., Téllez Vázquez, Y. \& López Ramírez, J. (2013) Tendencias de la Migración Interna. p.90-91. Recuperado de www.scielo.org.mx/scielo.php?pid=S14057425 2013000200003

Rossmo, D. K. (2011). Evaluating geographic profiling. Crime Mapping: A journal of research and practice, 3, 42-65.

Rossmo, D. K. (2014). Geographic profiling. En G. Bruinsma y D. Weisburd (Eds.), Encyclopedia of criminology and criminal justice (pp. 1934-1942). Nueva York: Springer. International e-Journal of Criminal Science Artículo 3, Número 9 (2015) http://www.ehu.es/inecs ISSN: 1988-7949 24

Rossmo, K. (2005) Geographic Heuristics or Shortcuts to Failure?: Response to Snook et al. Applied Cognitive Psychology . Appl. Cognit. Psychol. 19: 651-654 (2005)

Rossmo, D. K. (1995): “Geographic Profiling: Target patterns of serial murderes". Simon Fraser University: Vancouver.

Rossmo, D. K. (2000). Geographic profiling. CRC Press.

Rossmo, D. K. (1995). Place, space, and police investigations: Hunting serial violent criminals. En D. Weisburd y J. E. Eck (Eds.), Crime and place. Crime prevention studies, vol. 4 (pp. 217-235). Monsey, NY: Criminal Justice Press.

Rossmo, D.K., y Summers, L. (2015). “El perfil geográfico en la investigación criminal. International eJournal of Criminal Science, Numero 9 (artículo 3).

Ruíz Hidalgo, Á. (2017) El turismo sexual: proyecto de investigación en la costa del sol, (Trabajo de Grado), Universidad de Jaén, Facultad de Ciencias Sociales y Jurídicas. En: http://tauja.ujaen.es/bitstream/10953.1/7106/1/lvaro_Ruiz_Hidalgo._EL_TURISMO_SEXUAL_EN_L A_COSTA_DEL_SOL._PROYECTO_DE_INVESTIGACIN.pdf 
Salafranca, D. (2016). “Criminología aplicada al Análisis Delictual”, 6to Taller de entrenamoiento sobre el análisis delictual. Santiago, 27 de julio. Disponible en: El perfil geográfico criminal una nueva propuesta Pag. 32 Revista GEOESPACIAL (2019) https://es.scribd.com/document/377055732/Taller-4Criminologia-Apli [Consultado: 13-02-2019].

Salcedo-Albaran, E.; Garay Salamanca, L. J. (2016), Macro-Criminalidad: Complejidad y Resiliencia de las Redes Criminales. A Vortex Fundation and Small Wars Journal -El Centro Book. En: https://books.google.com.mx/books?id=xhjLDAAAQBAJ\&dq=REDES+CRIMINALES\&lr=lang_es\&h l=es\&source=gbs_navlinks_s

Salt, J. y Stein, J. (1997), "Migration as a business: the case of trafficking”, en International Migration, Vol. 35 (4), OIM, Ginebra.

Sánchez Hernández, C. E. (2019), Viajes y turismo asociados a la explotación sexual comercial de niños, niñas y adolescentes en Colombia: pornografía infantil*, Revista IUSTA, núm. 51. Universidad Santo Tomás, Colombia. Disponible en: https://www.redalyc.org/articulo.oa?id=560360081006

Skrobanek, S. , Boonpakdi N.a, Janthakeero C. (1997), Tráfico de mujeres: realidades humanos en el negocio internacional del sexo, Nancea, S.A. de ediciones, España.

Tiffon Nonis, Bernat-Noël (2021), Personalidad criminal en un militar de élite entrenado y asesinato. South Florida Journal of Development, Miami, v.2, n.4, p. 5531-5536 jul./sep. 2021.

United Nations General Assembly (2000), Report of the Ad Hoc committee on the Elaboration of a convention against Transnational Organized Crime on the work of its first to eleventh sessions, Annex I, II and III, -/55/383, ONU, Nueva York.

Vaca Cortés, J., y Aguilar, P., D. (2012). La máscara del asesino. Universidad Autónoma de Yucatán, México. 